\title{
Community structure, population structure and topographical specialisation of Gyrodactylus (Monogenea) ectoparasites living on sympatric stickleback species
}

\author{
Joost A.M. Raeymaekers ${ }^{1}$, Tine Huyse ${ }^{1}$, Hannelore Maelfait ${ }^{1,2}$, Bart Hellemans ${ }^{1}$ and Filip A.M. Volckaert ${ }^{1}$ \\ ${ }^{1}$ Katholieke Universiteit Leuven, Laboratory of Animal Diversity and Systematics, Ch. Deberiotstraat 32, B-3000 Leuven, \\ Belgium; \\ ${ }^{2}$ Current address: Coordination Centre for Integrated Coastal Zone Management, Wandelaarkaai 7, B-8400 Oostende, Belgium
}

Key words: host specificity, coexistence, Gasterosteus aculeatus, Pungitius pungitius, Gyrodactylidae, host density, site specificity, sympatry

\begin{abstract}
In order to disentangle the contribution of host and parasite biology to host specificity, we compared the structure and population dynamics of the Gyrodactylus (von Nordmann, 1832) flatworm community living on sympatric three-spined Gasterosteus aculeatus L. and nine-spined Pungitius pungitius (L.) stickleback. Between April 2002 and March 2003, a small lowland creek was sampled monthly. Species identity of about $75 \%$ of the worms per host was determined with a genetic nuclear marker (ITS1). Each stickleback species hosted a characteristic gill- and fin-parasitic Gyrodactylus: G. arcuatus Bychowsky, 1933 and G. gasterostei Gläser, 1974 respectively infecting the three-spined stickleback, with G. rarus Wegener, 1910 and G. pungitii Malmberg, 1964 infecting the nine-spined stickleback. Host size and seasonal dynamics were strong determinants of parasite abundance. A strong interaction between host and parasite species determined infection levels and affected three levels of parasite organisation: community structure, population structure and topographical specialisation. Community and population structure were shaped by asymmetric cross-infections, resulting in a net transmission of the Gyrodactylus species typical of the nine-spined stickleback towards the three-spined stickleback. Host density was not a major determinant of parasite exchange. Aggregation and topographical specialisation of the Gyrodactylus species of the three-spined stickleback were more pronounced than that of the nine-spined stickleback.
\end{abstract}

The ecological context of the population dynamics of parasites is important to understand host-parasite systems. Epidemiological models predict a positive relationship between host population density and abundance of directly transmitted macroparasites (Arneberg et al. 1998, Arneberg 2001). Variation in parasite abundance may point to transmission rates relative to the probability of physical contact between hosts. The population dynamics of directly transmitted parasites may thus provide information on the interplay between parasite population structure, parasite behaviour, host ecology, and host behaviour.

A central aspect of this interaction is host specificity, which determines whether a parasite is able to colonise a host (Sasal et al. 1999a). Host specificity can be strongly determined by host ecology, such as the degree of host isolation, and by parasite ecology, such as mobility (ter Hofstede et al. 2004). This is reflected in the parasite population structure. For instance, the degree of physical contact between hosts affects the degree of parasite aggregation (Sasal et al. 1999b). In ectoparasites, the host and parasite components of host specificity may influence two additional levels of parasite organisation. First, host specificity may account for ecto- parasite community structure. For instance, specialist parasite communities may reveal a higher degree of nestedness than generalist parasite communities (Matějusová et al. 2000, Morand et al. 2002). Secondly, topographical specialisation of ectoparasites may be influenced by factors such as proximity to mates, competition, or host defence strategies (Reiczigel and Rózsa 1998, ter Hofstede et al. 2004).

The link between ectoparasite ecology and host ecology, and its consequences for parasite microhabitat, population and community structure seems evident, but it is hard to disentangle the host and parasite component of host specificity in the field. Inference may be particularly strong when sympatric host species share a community of closely related, host-specific ectoparasites, accounting for several replications of host-parasite systems in a single environment. The present study system consists of sympatric three-spined stickleback, Gasterosteus aculeatus L., and nine-spined stickleback, Pungitius pungitius (L.). Both fishes host several monogenean parasites of the genus Gyrodactylus (von Nordmann, 1832) (Malmberg 1970, Dartnall 1973, Wootton 1976, Cone and Wiles 1985, Harris 1985, Kalbe et al. 2002, Özer et al. 2004). Gyrodactylus species are often

Address for correspondence: J. Raeymaekers, Katholieke Universiteit Leuven, Laboratory of Animal Diversity and Systematics, Ch. Deberiotstraat 32, B-3000 Leuven, Belgium. Phone: ++32 16323 966; Fax: ++32 16324 575; E-mail: joost.raeymaekers@bio.kuleuven.be 
highly specific (Harris 1985, Poulin 1992, Bakke et al. 2002), and given their direct life cycle and direct transmission they are expected to depend heavily on host biology. The stickleback immune system can respond strongly a few weeks after infection (Lester and Adams 1974, Raeymaekers et al. 2006). Although transmission by direct host contact is not the only route of transmission (Bakke et al. 1992, 2007), transmission rates are probably correlated with the physical contact between hosts (Boeger et al. 2005).

The three-spined and the nine-spined stickleback can coexist and are closely related, but their biology differs considerably (Bråten 1966, Wootton 1976, 1984, Copp and Kováč 2003, Hart 2003). The same is true for their Gyrodactylus species, which differ markedly in host specificity, microhabitat specificity and reproductive strategy, although the latter is less well understood (Harris 1993). Our first objective was to determine the structure and seasonal dynamics of the Gyrodactylus communities and populations living on coexisting threeand nine-spined sticklebacks. The occurrence of four Gyrodactylus species, identified with molecular tools, was linked with host characteristics. Secondly, we studied the site specificity of each Gyrodactylus species. Finally, three Gyrodactylus species in our study were recorded on both stickleback species, with a higher occurrence on the "primary host" species compared to the "secondary host" species. Ratios of infection success in primary versus secondary host species enabled us to compare the contribution of host and parasite components to host specificity. We discuss the implications at three levels of parasite organisation: community structure, population structure, and topographical specialisation (sensu Pie et al. 2006).

\section{MATERIALS AND METHODS}

Sampling and molecular identification. The study was carried out in a small eutrophic polder creek located in Westkerke (Belgium; $\left.51^{\circ} 10^{\prime} \mathrm{N}, 3^{\circ} 00^{\prime} \mathrm{E}\right)$. The site is located near the North Sea coast, and has a very slow current of freshwater and a high density of macrophytes. Between April 2002 and March 2003, we sampled this site monthly for 25-30 individuals of three-spined and nine-spined stickleback each. Water temperature $\left({ }^{\circ} \mathrm{C}\right)$ and conductivity $\left(\mu \mathrm{S} . \mathrm{cm}^{-1}\right)$ were monitored with a probe (Hach, Loveland, Co, USA). Both stickleback species were present throughout the year along a stretch of $300 \mathrm{~m}$. An estimate of the proportion of nine-spined versus three-spined sticklebacks along this stretch was determined by sampling during $30 \mathrm{~min}$ in March 2003; nine-spined $(\mathrm{n}=87)$ and three-spined sticklebacks $(n=124)$ were present in a ratio of 0.70 . Other fish species were rare. Dipnetting along $50 \mathrm{~m}$ of the stretch was usually sufficient to obtain 30 sticklebacks of each species. To avoid an aggregation bias, individuals were selected randomly when more than one stickleback was caught in one dip. As a result of water turbidity and plant growth, all captures were blind. After capture, sticklebacks were individually preserved in $100 \%$ ethanol. In the labora- tory, each fish was measured (standard length, SL \pm 0.1 $\mathrm{cm})$, weighed ( $\pm 0.01 \mathrm{~g})$, inspected for Gyrodactylus sp. in a Petri-dish under a stereomicroscope, and dissected for sex determination when larger than $25 \mathrm{~mm}$. All worms were counted on each of the following fish body parts: caudal fin (cf), anal fin (af), dorsal fin including spines (df), pelvic plate and pelvic spines (pf), left and right pectoral fin (pc), head including the throat and the surface of the eyes (h), and the rest of the body (b) and gills (g). Gill covers were removed in order to dissect and inspect the gills. Detached worms were counted separately.

Worms were collected with a dissection needle and transferred to a $250 \mu \mathrm{l}$ Eppendorf tube containing $5 \mu \mathrm{l}$ Milli-Q water. All worms were collected whenever possible. In case of high infection numbers, parasites were sampled proportionally to the distribution over the body, with a maximum of 50 worms per fish. Eppendorf tubes were stored at $-20^{\circ} \mathrm{C}$ until further processing. Samples were digested by the addition of 5 $\mu 1$ of a double concentrated lysis solution, at a final concentration of $1 \times$ PCR buffer (Eurogentec), $0.45 \%$ Tween 20 (Merck, Germany), 0.45\% NP40 (Calbiochem, USA) and 60 $\mu \mathrm{g} \mathrm{l}^{-1}$ proteinase K (Sigma, USA). Tubes were centrifuged, incubated at $65^{\circ} \mathrm{C}$ for $25 \mathrm{~min}$, followed by incubation at $95^{\circ} \mathrm{C}$ for $10 \mathrm{~min}$.

Identification of the worms was based on the Internal Transcribed Spacer 1 (ITS1) rDNA region. The $20 \mu 1$ PCR contained $2 \mu$ l genomic DNA, $1 \times$ PCR buffer (Eurogentec), 1.25 $\mathrm{mM} \mathrm{MgCl} 2,200 \mu \mathrm{M}$ of dNTP, $1 \mu \mathrm{M}$ ITS1f (Cunningham 1997), $1 \mu \mathrm{M}$ ITS1r (Zietara et al. 2002), 1 U of Silver Taq Polymerase (Eurogentec) and Milli-Q water. Samples were subjected to an initial denaturation step of $5 \mathrm{~min}$ at $95^{\circ} \mathrm{C}$, followed by 40 cycles of $30 \mathrm{~s}$ at $95^{\circ} \mathrm{C}, 30 \mathrm{~s}$ at $54^{\circ} \mathrm{C}$ and $30 \mathrm{~s}$ at $72^{\circ} \mathrm{C}$, with a final elongation step of $7 \mathrm{~min}$ at $72^{\circ} \mathrm{C}$. Aliquots $(5 \mu 1)$ of PCR products were run on a $1 \%$ agarose gel before and after digestion with $5 \mathrm{U}$ of restriction enzymes (RE). RE were selected based on all available ITS sequences from $G$. arcuatus Bychowsky, 1933, G. rarus Wegener, 1910, G. branchicus, Malmberg, 1964, G. pungitii Malmberg, 1964, and G. gasterostei Gläser, 1974 submitted to GenBank (AJ001839, AJ001841, AJ001845, AF156668, AF156669, AF 484543, AF328865, AF328867, AF328869, AY338442, AY338443, AY338444, AY338445, AY061976). RE Bst $1107 \mathrm{I}$ was used to discriminate between G. gasterostei and G. pungitii. The ITS1 fragment, about 770 bp long, digested into two fragments of 303 and $467 \mathrm{bp}$ in the case of G. pungitii, and no restriction in the case of G. gasterostei. Each digestion and electrophoresis step included a positive control sample of $G$. pungitii to check the quality of the enzyme. RE BseNI was used to discriminate between G. rarus, G. arcuatus and $G$. branchicus. The enzyme cuts the 498 bp ITS1 fragment into a fragment of $328 \mathrm{bp}$ and $170 \mathrm{bp}$ in G. rarus, and into a fragment of 294 and 224 bp in G. arcuatus. Specimens of G. branchicus, lacking a BseNI restriction site (Zietara et al. 2000), were not observed. However, a more recent GenBank ITS1 submission for G. branchicus from Finland (AY061977; Zietara and Lumme 2003) contains the same restriction site as $G$. rarus. The identification of $G$. rarus was therefore confirmed by direct sequencing of the ITS1 fragment of 49 specimens using Big Dye version 1.1 on an Applied Biosystems 3130 DNA Analyser. 
Data analysis. First, gyrodactylid identification success and gyrodactylid infracommunity structure were determined. Secondly, we investigated for each Gyrodactylus species to what extent two determinants of population structure, prevalence and infection intensity, differed between host species, host sex, host size and sampling month. As parasite species level could not always be determined for the complete Gyrodactylus fauna of an individual host, infection intensities of a particular parasite species may represent an underestimation for that particular host. Therefore, we allocated unidentified worms to a particular parasite species proportional to the overall occurrence of that parasite species in the whole sample of hosts. Overall occurrence was calculated as the number of worms of a species divided by the total number of identified worms, and this was done in each host species separately. From the corrected infection intensity we calculated corrected prevalence, which slightly exceeded uncorrected values. Prevalence, mean and median intensities were compared among subsets of hosts as implemented in the software QP 3.0 (Rózsa et al. 2000). Third, we analyzed the topographical specialisation (Pie et al. 2006) of each parasite species in relation to host species and host sex. Significance of the association between rows and columns of the contingency tables was based on $\chi^{2}$ values calculated and tested in STATISTICA 6.0. Finally, we studied the changes in topographical specialisation associated with increasing infection intensity.

\section{RESULTS}

\section{Habitat and sticklebacks}

Mean monthly temperatures varied between $18.8^{\circ} \mathrm{C}$ in August and $3.6^{\circ} \mathrm{C}$ in January (Fig. 1a). Size was largest in April for both the three-spined $(4.45 \pm 0.37 \mathrm{~cm})$ and the nine-spined $(4.15 \pm 0.39 \mathrm{~cm})$ sticklebacks. Sizes dropped steeply to minima in July for the three-spined stickleback $(1.94 \pm 0.43 \mathrm{~cm})$ and in August for the ninespined stickleback $(2.35 \pm 0.48 \mathrm{~cm})$, due to the emergence of juveniles $(<25 \mathrm{~mm})$. Adults of the three-spined stickleback were almost absent in July and August, whereas adult nine-spined stickleback persisted in reasonable numbers during summer. This period coincided with the growth of a dense layer of duck weed covering almost the entire water body. From October onwards, most young of the year had grown larger than $30 \mathrm{~mm}$. Sex-ratios were biased towards females (female:male ratio in three-spined stickleback: $1.45: 1$; in nine-spined stickleback: 1.28:1); males were particularly rare in the three-spined stickleback in late autumn. The threespined stickleback sample consisted all year round of a mix of highly, partially and low-plated individuals, which is typical for the lowland populations bordering the North Sea (Raeymaekers et al. 2005, 2007).

\section{Gyrodactylus parasitology, species and community structure}

Out of a total of 603 fish, 348 were infected with 4,011 Gyrodactylus sp. About one third of the worms
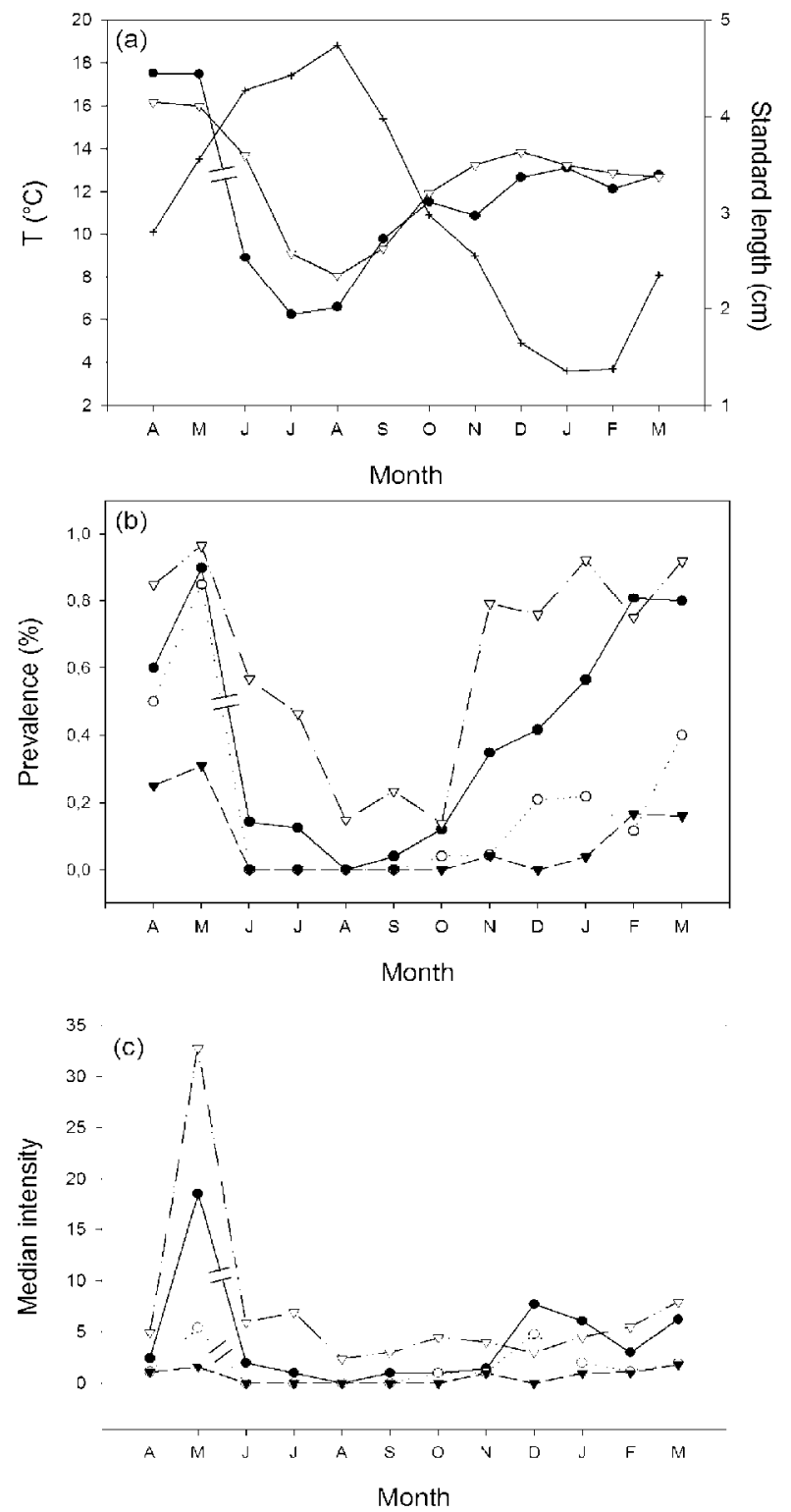

Fig. 1. Dynamics of two coexisting stickleback species and their Gyrodactylus parasites from a small polder creek in Westkerke (Belgium) between April 2002 and March 2003. (a) Average standard length $(\mathrm{cm})$ of nine-spined (triangles) and three-spined (circles) sticklebacks along with average monthly temperatures $\left({ }^{\circ} \mathrm{C}\right.$; cross marks). (b) Prevalence and (c) median intensity of $G$. pungitii (open symbols) and $G$. gasterostei (black symbols) in nine-spined (triangles) and three-spined (circles) stickleback. The breakpoint in the graphs between May and June represents the disappearance of the adult cohort of three-spined sticklebacks.

$(32 \%)$ was not sampled, whereas the DNA amplification success was $83 \%$. As a result, $45 \%$ of the worms could not be identified, but the average fraction of identified worms per host was $0.75 \pm 0.30$ (median: 0.87). There was no difference in identification success of worms between host species (Mann-Whitney U test; $\mathrm{P}=$ 

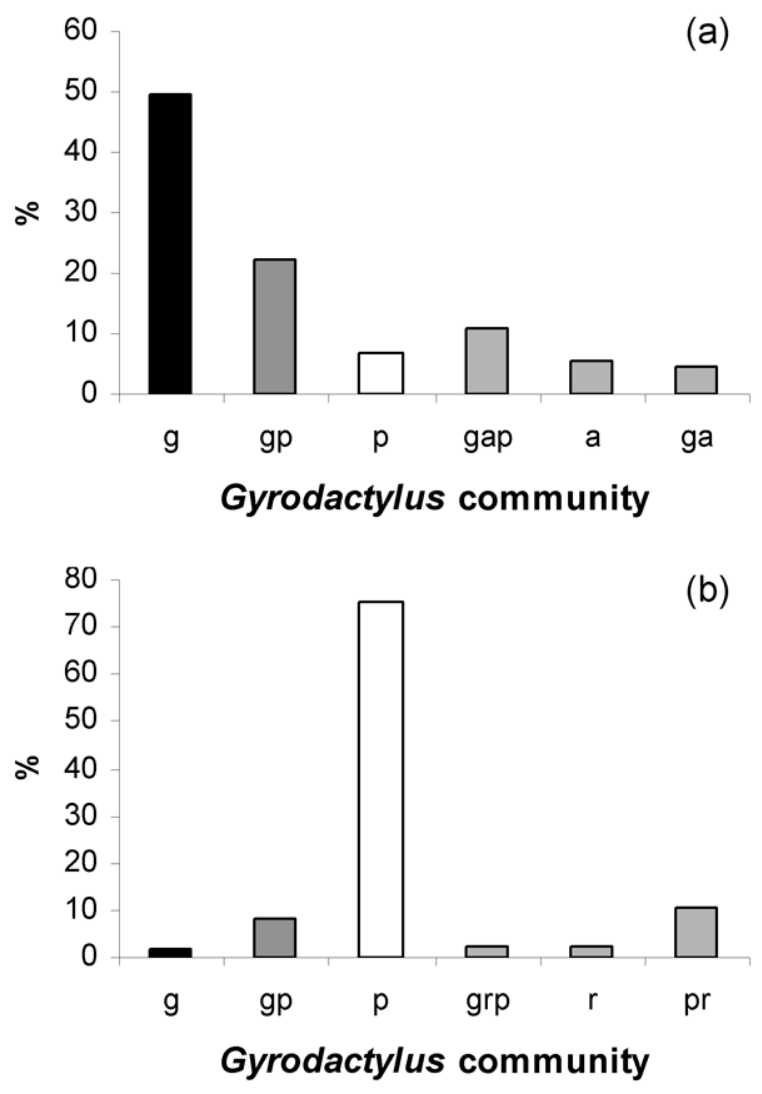

Fig. 2. Composition (\%) of Gyrodactylus communities on (a) three-spined $(n=129)$ and $(\mathbf{b})$ nine-spined $(n=201)$ stickleback from a small polder creek in Westkerke (Belgium) between April 2002 and March 2003. Abbreviations: a - G. arcuatus; $\mathrm{g}-$ G. gasterostei; $\mathrm{p}-\mathrm{G}$. pungitii; $\mathrm{r}-$ G. rarus. Other classes (gp, gap, ga, grp and pr) represent multispecies communities.

0.75). Prevalence in three-spined stickleback (48.6\% of 286 hosts) was considerably lower compared to ninespined stickleback $(65.9 \%$ of 317 hosts $)\left(\chi^{2}=18.5 ; \mathrm{P}\right.$ $<0.0001)$. Median intensities were also lower in the three-spined stickleback (i.e. 3 versus 6 worms per fish; Mood's median test; $\mathrm{P}=0.038$ ), whereas mean intensities were not significantly different $(\mathrm{t}=0.547 ; \mathrm{P}=$ 0.581 ): the distribution of worms on three-spined stickleback was much more overdispersed due to three massinfestations of 130 to 275 worms. The highest infection level found on nine-spined sticklebacks was 76 worms.

Gyrodactylus individuals detected on three-spined stickleback ( $\mathrm{n}=802$ identified worms) belonged to $G$. gasterostei (81.7\%), Gyrodactylus pungitii (13.2\%), G. arcuatus $(4.2 \%)$ and $G$. rarus $(0.9 \%)$. Gyrodactylus pungitii $(93.6 \%)$, G. rarus (3.8\%) and G. gasterostei $(2.6 \%)$ were found on the nine-spined stickleback $(\mathrm{n}=$ 1,422 identified worms). Gyrodactylus pungitii and $G$. gasterostei are considered fin or skin parasites, whereas G. arcuatus and G. rarus are mainly encountered on gills (Malmberg 1970). About 49\% of the Gyrodactylus communities on the three-spined stickleback consisted exclusively of G. gasterostei (Fig. 2). Furthermore, 29\% of the three-spined sticklebacks hosted exclusively $G$. pungitii or G. pungitii combined with G. gasterostei, whereas $10 \%$ were infected with $G$. arcuatus with or without G. gasterostei. Only $11 \%$ were infected with all three Gyrodactylus species. On the nine-spined stickleback, $76 \%$ of the Gyrodactylus communities consisted exclusively of $G$. pungitii. Only $9 \%$ hosted $G$. gasterostei with or without G. pungitii, whereas $13 \%$ of the nine-spined sticklebacks were infected with $G$. rarus or G. rarus together with G. pungitii. The three Gyrodactylus species co-occurred in only $2 \%$ of the communities.

\section{Gyrodactylus population structure}

Host specificity and seasonal dynamics. The "gill" parasites, G. arcuatus and G. rarus, were only occasionally encountered, with a prevalence of less than $10 \%$ (Table 1). Gyrodactylus arcuatus was mostly found in late autumn on or close to the gills. Occasionally it was also present in other seasons and on other parts of the body, but exclusively on three-spined sticklebacks. Gyrodactylus rarus occurred throughout the year, mainly on the gills of the nine-spined stickleback, with a subtle peak in summer. Very rarely (4 out of 286 hosts) it was observed on the gills of the three-spined stickleback. Prevalence, mean and median intensity of both gill parasites on their respective primary hosts were similar $(\mathrm{P}>0.05$; Table 1). Indices of discrepancy (Poulin 1993), expressing how aggregated parasites are distributed over their hosts, were mostly greater for gill parasites than for fin parasites.

As suggested by their scientific names, both fin parasites, G. pungitii and G. gasterostei, occurred abundantly on their primary host species, the nine-spined and the three-spined stickleback, respectively (Table 1). Their presence was characterized by strong seasonal fluctuations. Prevalence (Fig. 1b) and median intensity (Fig. 1c) of both Gyrodactylus species peaked in May, but decreased considerably in summer, in parallel with the decrease in the number of adult fish. Gyrodactylus gasterostei virtually disappeared in August, when the three-spined stickleback population mainly consisted of juveniles. Intensities of G. gasterostei remained low until December, whereas prevalence was only fully recovered by February. For G. pungitii, the reduction in population size during summer was less pronounced, due to a persisting proportion of infected adult ninespined stickleback. Prevalence re-established quickly between October and November. Infection intensities of both fin parasites remained relatively stable during winter and early spring.

The fin parasites G. pungitii and G. gasterostei also cross-infected each others' primary host species as "secondary" hosts (Table 1). However, the signal of host 
specificity was strong, as confirmed by signifi- cant

Table 1. Total number of hosts $\left(n_{t}\right)$, number of infected hosts $\left(n_{i}\right)$, prevalence $\left(n_{i} / n_{t}\right)$, mean intensity, median intensity and index of discrepancy (D; Poulin 1993) for four Gyrodactylus species infecting three-spined (Gasterosteus aculeatus) and nine-spined (Pungitius pungitius) stickleback in a small polder creek in Westkerke (Belgium) between April 2002 and March 2003. P/S indicates whether the stickleback species is a primary $(\mathrm{P})$ or a secondary $(\mathrm{S})$ host for the parasite. Infection parameters are based on values extrapolated towards total number of worms per host. F - fin and skin parasite; G - gill parasite.

\begin{tabular}{|c|c|c|c|c|c|c|c|c|}
\hline Parasite & Host & $\mathrm{P} / \mathrm{S}$ & $\mathrm{n}_{\mathrm{t}}$ & $\mathrm{n}_{\mathrm{i}}$ & $\mathrm{n}_{\mathrm{i}} / \mathrm{n}_{\mathrm{t}}$ & Mean intensity & Median intensity & $\mathrm{D}$ \\
\hline G. gasterostei $(\mathrm{F})$ & G. aculeatus & $\mathrm{P}$ & 286 & 113 & $39.5 \%$ & 12.40 & 4.0 & 0.875 \\
\hline G. pungitii (F) & G. aculeatus & S & 286 & 52 & $18.2 \%$ & 4.18 & 2.1 & 0.915 \\
\hline G. $\operatorname{arcuatus}(\mathrm{G})$ & G. aculeatus & $\mathrm{P}$ & 286 & 27 & $9.4 \%$ & 2.44 & 1.2 & 0.945 \\
\hline G. gasterostei (F) & P. pungitius & $\mathrm{S}$ & 317 & 24 & $7.6 \%$ & 1.88 & 1.3 & 0.948 \\
\hline G. pungitii (F) & P. pungitius & $\mathrm{P}$ & 317 & 193 & $60.9 \%$ & 11.03 & 5.9 & 0.719 \\
\hline G. rarus $(\mathrm{G})$ & P. pungitius & $\mathrm{P}$ & 317 & 31 & $9.8 \%$ & 2.10 & 1.2 & 0.937 \\
\hline
\end{tabular}

Table 2. Prevalence (\%) of four Gyrodactylus species on four size classes of three-spined (Gasterosteus aculeatus) and ninespined (Pungitius pungitius) stickleback in a small polder creek in Westkerke (Belgium) between April 2002 and March 2003.

\begin{tabular}{lrlcccc}
\hline Host & $\mathrm{n}$ & Host size $(\mathrm{cm})$ & G. arcuatus & G. rarus & G. gasterostei & G. pungitii \\
\hline G. aculeatus & 65 & $<2.5$ & 6.2 & - & 9.2 & 0 \\
& 127 & $2.5-3.4$ & 5.5 & - & 34.6 & 11.8 \\
& 74 & $3.5-4.4$ & 14.9 & - & 63.5 & 29.7 \\
& 20 & $>4.5$ & 25.0 & - & 80.0 & 75.0 \\
\hline P. pungitius & 51 & $<2.5$ & - & 5.9 & 0 & 21.6 \\
& 122 & $2.5-3.4$ & - & 7.4 & 5.7 & 52.5 \\
& 127 & $3.5-4.4$ & - & 13.4 & 10.2 & 81.1 \\
& 17 & $>4.5$ & - & 11.8 & 23.5 & 88.2 \\
\hline
\end{tabular}

differences between primary and secondary host species in terms of prevalence $(\mathrm{P}<0.0001)$ and median infection intensity $(\mathrm{P}<0.001)$. Moreover, on its primary host, each parasite species outperformed the other in terms of prevalence $(\mathrm{P}<0.0001)$. The index of discrepancy was higher in the infected secondary host species (Table 1). Seasonal fluctuations of prevalence and median infection intensity on secondary host combinations resembled primary host combinations, albeit at lower levels (Fig. 1b, c). Interestingly, host specificity was also asymmetric: ratios of prevalence and intensity on primary versus secondary host species indicated that the three-spined stickleback is more important as a secondary host species for $G$. pungitii than the nine-spined stickleback for G. gasterostei (Table 1). Gyrodactylus pungitii was also not significantly underrepresented on the three-spined stickleback compared to G. gasterostei in terms of median intensity $(\mathrm{P}=0.132)$. Conversely, $G$. gasterostei was underrepresented on the nine-spined stickleback compared to G. pungitii $(\mathrm{P}<0.0001)$.

In March 2003, the prevalence of G. gasterostei was lower on three-spined stickleback $(80 \%)$ compared to G. pungitii on nine-spined stickleback $(92 \%)$, despite the higher density of three-spined versus nine-spined stickleback. In the same month, the ratio of prevalences on secondary hosts $(0.40$; i.e. $16 \%$ for G. gasterostei in nine-spined stickleback, divided by $40 \%$ for $G$. pungitii in three-spined stickleback) was much smaller than the estimated host density ratio $(0.70)$, which is an estimate (assuming random mixing) of the odds for a worm encountering nine-spined versus three-spined stickleback.
These results suggest that host density is not the major determinant of the transmission probabilities of worms within and among host species.

Host sex and host size. Male and female stickleback displayed no differences in prevalence, mean or median intensity for any of the four Gyrodactylus species, except for G. pungitii, which was more prevalent in male nine-spined stickleback (males: $78 \%$; females: $62 \%$; $\mathrm{P}$ $=0.005)$. The difference was particularly striking in July, the last month of the breeding season in ninespined stickleback (Copp and Kováč 2003). Across all host-parasite species combinations, prevalence strongly increased with host size (Table 2), with median intensity being minimal in the juvenile size class $(<25 \mathrm{~mm})$. Up to $22 \%$ and $6 \%$ of the juveniles were infected with their primary fin and gill parasites, respectively. Juveniles did not serve as secondary hosts.

\section{Topographical specialisation}

Gyrodactylus communities were differentially distributed over the body of both stickleback species $\left(\chi^{2}=\right.$ 343.12 ; df $=7$; $\mathrm{P}<0.0001$; Fig. 3 ). Worms on threespined stickleback (with a majority of G. gasterostei) had a greater affinity for the head and the pelvic plate and spines. On nine-spined stickleback (with a majority of G. pungitii), the dorsal and anal fin were more preferred, and almost no worms were found on the pelvic plate and spines. The proportional occurrence on pectoral fins, caudal fin and other body parts was similar between host species. Within host species, toporaph- 

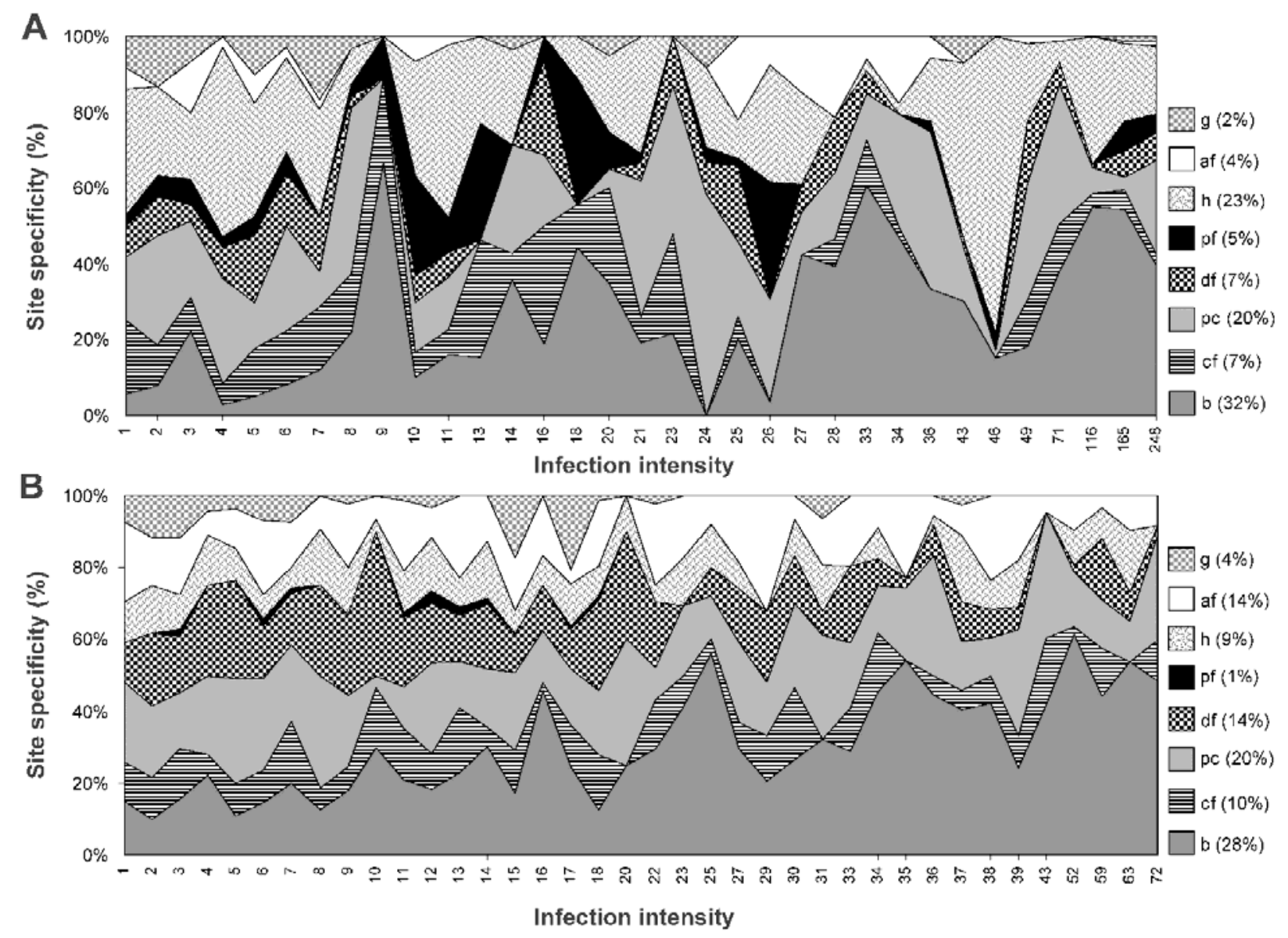

Fig. 3. Topographical specialisation of Gyrodactylus communities at increasing intensities on $(\mathbf{A})$ three-spined $(\mathrm{n}=137)$ and $(\mathbf{B})$ nine-spined $(\mathrm{n}=206)$ stickleback from a small polder creek in Westkerke (Belgium) between April 2002 and March 2003. Abbreviations: af - anal fin; $\mathrm{b}$ - body; $\mathrm{cf}$ - caudal fin; $\mathrm{df}$ - dorsal fin and dorsal spines; $\mathrm{g}$ - gills; $\mathrm{h}$ - head; $\mathrm{pc}$ - pectoral fins; $\mathrm{pf}-$ pelvic plate and pelvic spines.

ical specialisation differed between males and females (three-spined stickleback: $\chi^{2}=39.48$; df $=7$; $\mathrm{P}<0.0001$; nine-spined stickleback: $\left.\chi^{2}=51.94 ; \mathrm{df}=7 ; \mathrm{P}<0.0001\right)$. We observed a similar shift in both species, with worms being proportionally more predominant on fins in males and more on the head and other parts of the body in females.

With increasing infection intensities, worms on ninespined stickleback were proportionally more often encountered on the body of the fish (Fig. 3). Remarkably, this was not the case for worms on three-spined stickleback: topographical specialisation in function of infection intensity changed in a highly irregular way including occasional site emptiness. On some three-spined sticklebacks, worms aggregated at more specific sites like the head and the pectoral fins. As a result, worms on nine-spined stickleback were displaying a more diverse distribution over sites, as revealed by the Simpson index (on nine-spined stickleback: $2.65 \pm 1.20$; on three-spined stickleback: $2.24 \pm 1.19 ; \mathrm{P}=0.0019$ ).

Topographical specialisation of $G$. pungitii on threespined stickleback deviated significantly from its distri- bution on nine-spined stickleback $\left(\chi^{2}=33.03\right.$; df $=7$; $\mathrm{P}<0.0001)$. On three-spined stickleback, G. pungitii maintained its preference for the anal fin, but showed a greater affinity for the pelvic plate and spines, and weaker for the dorsal fin, similar to G. gasterostei. For G. gasterostei, topographical specialisation depended only marginally on host species $\left(\chi^{2}=13.52\right.$; $\mathrm{df}=7 ; \mathrm{P}=$ 0.06). On nine-spined stickleback, G. gasterostei showed an increased preference for the head and the pectoral fins, but was absent from the pelvic plate and spines.

\section{DISCUSSION}

We studied the dynamics of the Gyrodactylus fauna living on sympatric stickleback species from a small polder creek in Belgium. We document community structure, population structure, host specificity and topographical specialisation of four Gyrodactylus species in the course of a single year. Whereas we cannot derive general conclusions like those obtained in multi-year and multi-site studies (e.g. Harris 1993), the identifica- 
tion of a high number of worms with molecular tools generated great detail on local gyrodactylid population and community biology.

\section{Gyrodactylus community structure}

The Gyrodactylus community of both stickleback species in the Westkerke polder was dominated by a specific gill parasite and a specific fin parasite: G. arcuatus and G. gasterostei on the three-spined stickleback, and G. rarus and G. pungitii on the nine-spined stickleback. Occurrence, host specificity and topographical specialisation of each parasite largely agreed with previous field studies on sticklebacks (Malmberg 1970, Harris 1985, Özer et al. 2004). For euryhaline fish like sticklebacks, Gyrodactylus community structure may largely reflect the environment. Both fin parasites represented more than $90 \%$ and $80 \%$ of the Gyrodactylus community of nine- and three-spined sticklebacks, respectively. The occurrence of the euryhaline gill parasites ( $G$. arcuatus and $G$. rarus) was relatively low. For G. rarus, this has been reported earlier by Malmberg (1970). In contrast, a prevalence of more than $80 \%$ has been described for G. arcuatus infecting the three-spined stickleback in a euryhaline stream at the mouth of the Black Sea (Özer et al. 2004). An extremely localised autumn outbreak of $G$. arcuatus has been documented in river sticklebacks by Harris (1993). Bakke et al. (2002, 2007) consider G. arcuatus as a species with a wide host range and efficient dispersal, but poor competitive ability, leading to rapid exclusion from fully developed communities. However, the role of competition remains controversial in ectoparasites; there is little evidence that parasite populations or communities are structured by resource limitation (Rohde 1991, Reiczigel and Rózsa 1998, Morand et al. 2002, but see Pie et al. 2006).

There is little agreement on which other factors structure ectoparasite assemblages (Rohde 1991). It is hypothesized that host density, reflecting the probability of encountering a host, promotes abundance and species richness in communities of directly transmitted parasites (Arneberg et al. 1998, Arneberg 2001, 2002). This way host density may have contributed to the "natural ecological transfer" (King and Cable 2007) of the parasite species of the nine-spined stickleback towards the threespined stickleback. However, in at least one month we observed that the host with the lowest density (the ninespined stickleback) was more heavily infected. This suggests either that host density may be no major determinant of parasite exchange in this study, or that density estimates do not accurately predict host enctnotbes. hypothesis is that individual host size relates to infracommunity size (Guégan and Hugueny 1994, Matějusová et al. 2000). We found that juveniles were only infected by their typical parasites, such that infracommunities represented subsets of the infracommunities on adult hosts. The increase in species richness with host size therefore followed a nested pattern. The most likely cause of nestedness is differential colonisation (Worthen and Rohde 1996, Rohde et al. 1998). The chances of infection with Gyrodactylus may increase with host age or reproductive investment, although rapid acquisition of Gyrodactylus and other ectoparasites has been demonstrated in fry of brooding fish like sticklebacks, presumably through adult-fry interactions (Bakke et al. 2007). Interestingly, the proportion of secondary versus primary infections increased with host size as well.

\section{Gyrodactylus population structure}

Overall prevalence and infection intensity in ninespined stickleback was higher than in three-spined stickleback. Parasite abundance differed among parasite species and was strongly influenced by host size (see below). The parasite fauna was more strongly aggregated on three-spined stickleback than on nine-spined stickleback. Aggregation also differed between fin versus gill parasites, and between primary and secondary fin parasites on the same host. Aggregation, which can be considered to reflect parasite isolation (Sasal et al. 1999b), therefore seems to depend on the host species as well as on the parasite species.

Seasonal dynamics. Major factors affecting the seasonal prevalence and abundance of gyrodactylids include abiotic factors like water temperature, and hostrelated factors, such as behaviour, sex, age, resistance and mortality (Malmberg 1970, Bakke et al. 2002, Özer et al. 2004). The seasonal dynamics of Gyrodactylus parasitizing salmonids and marine gobies were characterized by increased population growth with rising temperatures in spring, peak reproduction in late summer, and declining reproductive rates in winter (Appleby 1996, Geets et al. 1998, Bakke et al. 2002). In several permanent freshwater fishes, prevalence in summer was low (Matějusová et al. 2000, Dávidová et al. 2005). In this study, Gyrodactylus intensity and prevalence on three-spined and nine-spined stickleback peaked in late spring, collapsed in early summer, remained low in autumn and were built up again during winter. Prevalence also followed the dynamics of average host size. The abrupt decline in early summer coincided with the end of the breeding season of both host populations, most likely due to mortality or migration of the postspawners. The decline occurred earlier and was stronger in three-spined stickleback. Survival of post-spawners may be higher in nine-spined sticklebacks, which are more resistant to oxygen depletion (Wootton 1984, Copp and Kováč 2003).

In October, prevalence was equally low in both stickleback species. The subsequent recovery of prevalence was much faster in nine-spined stickleback. This suggests that $G$. pungitii had a larger total population size than G. gasterostei at the onset of the recovery, or that the species has a greater growth rate or transmission 
success. The host's lifestyle may also promote the recovery, through shoaling behaviour (Boeger et al. 2005), the sediment (Bakke et al. 1992), or the vegetation. Microhabitat partitioning among both stickleback species has been demonstrated experimentally (Hart 2003) and in shallow streams in the field (Copp and Kováč 2003): three-spined stickleback has a more benthic life style, whereas nine-spined stickleback prefers to live in the vegetation. As both life styles may favour parasite transmission, the contribution of host biology to the observed differences remains unclear. Dead hosts can also represent a source for parasite transmission (Olstad et al. 2006). It has been suggested that $G$. gasterostei and G. pungitii employ different transmission strategies with respect to dead hosts: $G$. pungitii is thought to remain on a dead host (Malmberg 1970), whereas G. gasterostei leaves the host shortly after its death (Bakke et al. 2007).

Host specificity, host size and host sex. The typical parasites of the three-spined stickleback, G. arcuatus and G. gasterostei, were absent or relatively uncommon on the nine-spined stickleback. Conversely, typical species of the nine-spined stickleback, i.e. some G. rarus and a considerable fraction of G. pungitii, cross-infected three-spined stickleback. The fact that we observed this pattern for a pair of fin and a pair of gill parasites suggests an effect of host density, which was considerably higher for three-spined stickleback in at least one sampling month. However, comparison of infection levels of G. gasterostei and G. pungitii suggests that the biology of the parasite (prevalence, transmission capacity or reproductive success) is a stronger determinant of host specificity than host density. The shift of infections towards larger size classes suggests that infection and host switching represent a probabilistic process becoming more likely with host age.

A combination of factors, such as infection rate, survival, reproduction, population growth and virulence, determine host optimality (King and Cable 2007). Mean intensity and prevalence of $G$. pungitii doubled that of G. gasterostei on their respective secondary hosts. One explanation is the high prevalence of $G$. pungitii which may enhance natural ecological transfers. The optimality of each fish species as a host for G. pungitii has not been studied. The epidermis of the nine-spined stickleback appeared not to be suitable as a long-term food source for G. gasterostei (Bakke et al. 2002). This is consistent with the poor performance of G. gasterostei on nine-spined stickleback in this study. Our results also confirm previous studies on G. gasterostei populations from Western Europe reporting narrow host specificity and a strong affinity to the biology of the three-spined stickleback (Bakke et al. 2007). At least in Great Britain and Belgium, G. gasterostei could therefore be regarded as a strict specialist species.
Male nine-spined sticklebacks were more frequently infected than females during the breeding season, which may be due to behavioural or endocrinological changes. More frequent and more severe infections with Gyrodactylus spp. have been reported in mature males of brown trout (Pickering and Christie 1980), and in female stone loach in periods of reproductive investment (Šimková et al. 2005). Appleby (1996) and Geets et al. (1998) reported a close affinity between Gyrodactylus infestations and the breeding biology of two goby species, mediated by host-sediment and host-host contact. Male gobies building nests were more infected than females in the beginning of the spawning season, which is expected given the intensity of contact with the sediment. Later on females visiting these nests carried a higher parasite load. In August and September, we also observed that females from the nine-spined stickleback carried more worms than males, but this was supported by few observations. The lack of sex-related infection levels in the three-spined stickleback may relate to the shorter (Copp and Kováč 2003) and abrupt end of the breeding season in this species.

\section{Topographical specialisation}

In Monogenea, many factors have been implicated as determinants of topographical specialisation and its potential changes with infrapopulation size. These factors include accessibility to resources, parasite age and mating strategies, competition, age of infection, and transmission to new hosts (Pie et al. 2006, Bakke et al. 2007). In this study, both fin parasites preferred different sites on their respective primary host species, and each parasite shifted its distribution on its secondary host species. We also observed that $G$. pungitii maintains a diverse distribution over its hosts at any infection level, whereas G. gasterostei tends to aggregate. These results suggest that $G$. pungitii has a more flexible topographical specialisation than G. gasterostei. This higher mobility at the infrapopulation level appears to match with higher transmission rates at the population level. A causal relationship remains tentative at the moment.

\section{Conclusion}

In this field study, differential infection and crossinfection levels influenced three levels of parasite organisation: community structure, population structure and topographical specialisation. Parasites of the ninespined stickleback enriched the Gyrodactylus fauna of the three-spined stickleback. The typical parasites of the three-spined stickleback were more host-specific, showed a more aggregated distribution at the population level, and a more pronounced topographical specialisation at the infrapopulation level. Our study illustrates that similar Gyrodactylus species may exploit sympatric and closely related hosts in surprisingly different ways. 
The analysis of Gyrodactylus population and community biology with respect to host characteristics may reveal important ecological or behavioural interactions between hosts. Because of their direct life cycle and continuous need to find susceptible hosts, Gyrodactylus species strongly depend on host biology. Gyrodactylus may therefore represent a particularly useful system to study host-parasite evolutionary dynamics (Boeger et al. 2005, Decaestecker et al. 2007), and to assess the contribution of parasitism to the remarkable cases of stickleback speciation (McKinnon and Rundle 2002, Raeymaekers et al. 2007).
Acknowledgements. We thank S. Geldof, B. Christiaen, I. Hontis, N. Haumont and E. Descamps for field support and technical assistance. K.M. Wegner, M. Kalbe, E. Decaes-tecker and two anonymous referees provided valuable comments. J.A.M.R. received a $\mathrm{PhD}$ fellowship from the Fund for Scientific ResearchFlanders (FWO-Vlaanderen) and was kindly hosted by A.P. Hendry during the finalisation of this study. T.H. is a Postdoctoral Fellow of FWO-Vlaanderen. Research was sponsored by FWO-Vlaanderen (project G.0142.03)

\section{REFERENCES}

APPLEBY C. 1996: Population dynamics of Gyrodactylus sp. (Monogenea) infecting the sand goby in the Oslo Fjord, Norway. J. Fish Biol. 49: 402-410.

ARNEBERG P. 2001: An ecological law and its macroecological consequences as revealed by studies of relationships between host densities and parasite prevalence. Ecography 24: 352 358.

ARNEBERG P. 2002: Host population density and body mass as determinants of species richness in parasite communities: comparative analyses of directly transmitted nematodes of mammals. Ecography 25: 88-94.

ARNEBERG P., SKORPING A., GReNFell B., READ A.F. 1998: Host densities as determinants of abundance in parasite communities. Proc. R. Soc. Lond., B Biol. 265: 1283-1289.

BAKKE T.A., CABLE J., HARRIS P.D. 2007: The biology of gyrodactylid monogeneans: the "Russian-Doll Killers". Adv. Parasitol. 64: 161-376.

BAKKE T.A., HARRIS P.D., CABLE J. 2002: Host specificity dynamics: observations on gyrodactylid monogeneans. Int. J. Parasitol. 32: 281-308.

BAKKe T.A., Harris P.D., JANSEN P.A., HANSEN L.P. 1992: Host specificity and dispersal strategy in gyrodactylid monogeneans, with particular reference to Gyrodactylus salaris (Platyhelminthes, Monogenea). Dis. Aquat. Org. 13: 63-74.

BOeger W.A., KRITSKy D.C., PIE M.R., ENGers K.B. 2005: Mode of transmission, host switching, and escape from the Red Queen by viviparous gyrodactylids (Monogenoidea). J. Parasitol. 91: 1000-1007.

BRÅTEN T. 1966: Host specificity in Schistocephalus solidus. Parasitology 56: 657-664.

CONE D.K., WILES M. 1985: The systematics and zoogeography of Gyrodactylus species (Monogenea) parasitizing gasterosteid fishes in North America. Can. J. Zool. 63: 956960.

COPP G.H., KovÁČ V. 2003: Sympatry between threespine Gasterosteus aculeatus and ninespine Pungitius pungitius sticklebacks in English lowland streams. Ann. Zool. Fenn. 40: 341-355.

CUNNINGHAM C.O. 1997: Species variation within the Internal Transcribed Spacer (ITS) region of Gyrodactylus (Monogenea: Gyrodactylidae) ribosomal RNA genes. J. Parasitol. 83: 215-219.

DARTNALL H.J.G. 1973: Parasites of the nine-spined stickleback Pungitius pungitius (L). J. Fish Biol. 5: 505-509.

DÁVIDOVÁ M., JARKOVSKÝ J., MATĚJUSOVÁ I., GELNAR M. 2005: Seasonal occurrence and metrical variability of Gyrodactylus rhodei Žitňan 1964 (Monogenea, Gyrodactylidae). Parasitol. Res. 95: 398-405.
DeCAesteCKer E., GABA S., RAEYMAEKeRs J.A.M., StOKS R., VAn Kerckhoven L., Ebert D., De Meester L. 2007: Host-parasite 'Red Queen' dynamics archived in pond sediment. Nature 450: 870-873.

Geets A., Beuls I., Ollevier F. 1998: Population dynamics of Gyrodactylus sp. 1 (Monogenea) from two sympatric goby species, Pomatoschistus minutus and P. lozanoi (Gobiidae, Pisces), in the Grevelingen Voordelta Area (SW Netherlands). PhD thesis, K.U. Leuven, pp. 149-173.

GUÉGAN J.F., HUGUENY B. 1994: A nested parasite species subset pattern in tropical fish - host as major determinant of parasite infracommunity structure. Oecologia 100: 184-189.

HARRIS P.D. 1985: Species of Gyrodactylus von Nordmann, 1832 (Monogenea: Gyrodactylidae) from freshwater fishes in southern England, with a description of Gyrodactylus rogatensis sp. nov. from the bullhead Cottus gobio L. J. Nat. Hist. 19: 791-809.

HARRIS P.D. 1993: Interactions between reproduction and population biology in gyrodactylid monogeneans - a review. Bull. Fr. Pêche Piscic. 1: 47-65.

HART P.J.B. 2003: Habitat use and feeding behaviour in two closely related fish species, the three-spined and nine-spined stickleback: an experimental analysis. J. Anim. Ecol. 72: $777-$ 783.

Kalbe M., Wegner K.M., Reusch T.B.H. 2002: Dispersion patterns of parasites in $0+$ year three-spined sticklebacks: a cross population comparison. J. Fish Biol. 60: 1529-1542.

KING T.A., CABLE J. 2007: Experimental infections of the monogenean Gyrodactylus turnbulli indicate that it is not a strict specialist. Int. J. Parasitol. 37: 663-672.

LESTER R.J.G., ADAMS J.R. 1974: Gyrodactylus alexanderi: reproduction, mortality, and effect on its host Gasterosteus aculeatus. Can. J. Zool. 52: 827-833.

MALMBERG G. 1970: The excretory systems and the marginal hooks as basis for the systematics of Gyrodactylus (Trematoda, Monogenea). Ark. Zool. 2: 1-235.

MATĚJUSOVÁ I., Morand S., GelnAR M. 2000: Nestedness in assemblages of gyrodactylids (Monogenea: Gyrodactylidea) parasitising two species of cyprinid - with reference to generalists and specialists. Int. J. Parasitol. 30: 1153-1158.

MCKINNON J.S., RUNDLE H.D. 2002: Speciation in nature: the threespine stickleback model systems. Trends Ecol. Evol. 17: $480-488$.

MORAND S., ROHDE K., HAYWARD C. 2002: Order in ectoparasite communities of marine fish is explained by epidemiological processes. Parasitology 124: S57-S63.

OlstAD K., CABle J., ROBERTSEN G., BAKKE T.A. 2006: Unpredicted transmission strategy of Gyrodactylus salaris (Mono- 
genea: Gyrodactylidae): survival and infectivity of parasites on dead hosts. Parasitology 133: 33-41.

ÖZER A., OZTURK T., OZTURK M.O. 2004: Prevalence and intensity of Gyrodactylus arcuatus Bychowsky, 1933 (Monogenea) infestations on the three-spined stickleback, Gasterosteus aculeatus L., 1758. Turk. J. Vet. Anim. Sci. 28: 807-812.

PICKERING A.D., Christie P. 1980: Sexual differences in the incidence and severity of ectoparasitic infestation of the brown trout, Salmo trutta L. J. Fish Biol. 16: 669-683.

PIE M.R., ENGERS K.B., BoEger W.A. 2006: Density-dependent topographical specialisation in Gyrodactylus anisopharynx (Monogenoidea, Gyrodactylidae): boosting transmission or evading competition? J. Parasitol. 92: 459-463.

POULIN R. 1992: Determinants of host specificity in parasites of freshwater fishes. Int. J. Parasitol. 22: 753-758.

POULIN R. 1993: The disparity between observed and uniform distributions - a new look at parasite aggregation. Int. J. Parasitol. 23: 937-944.

RAEYMAEKERS J.A.M., MAES G.E., AUDENAERT E., VOLCKAERT F.A.M. 2005: Detecting Holocene divergence in the anadromous-freshwater three-spined stickleback (Gasterosteus aculeatus) system. Mol. Ecol. 14: 1001-1014.

RAEYMAEKERS J.A.M., VAN HOUDT J.K.J., LARMUSEAU M.H.D., GELDOF S., VOLCKAERT F.A.M. 2007: Divergent selection as revealed by $\mathrm{P}_{\mathrm{ST}}$ and QTL-based $\mathrm{F}_{\mathrm{ST}}$ in three-spined stickleback (Gasterosteus aculeatus) populations along a coastalinland gradient. Mol. Ecol. 16: 891-905.

RAEYMAeKers J.A.M., Wegner K.M., HuYse T., VOlCKaerT F.A.M. 2006: Infection dynamics of the monogenean parasite Gyrodactylus gasterostei on sympatric and allopatric host populations. PhD thesis, chapter IV. K.U. Leuven, pp. 83-96.

REICZIGEL J., RózSA L. 1998: Host-mediated site segregation of ectoparasites: an individual-based simulation study. J. Parasitol. 84: 491-498.

ROHDE K. 1991: Intra- and interspecific interactions in low density populations in resource-rich habitats. Oikos 60: 91-104.
ROHDE K., Worthen W.B., HeAP M., Hugueny B., GuÉGAN J.F. 1998: Nestedness in assemblages of metazoan ecto- and endoparasites of marine fish. Int. J. Parasitol. 28: 543-549.

RÓZSA L., REICZIGEL J., MAJOROS G. 2000: Quantifying parasites in samples of hosts. J. Parasitol. 86: 228-232.

SASAL P., NIQUIL N., BARTOLI P. 1999b: Community structure of digenean parasites of sparid and labrid fishes of the Mediterranean Sea: a new approach. Parasitology 119: 635-648.

Sasal P., Trouve S., Muller-Graf C., Morand S. 1999a: Specificity and host predictability: a comparative analysis among monogenean parasites of fish. J. Anim. Ecol. 68: 437444.

ŠImkovÁ A., JARkovskÝ J., KoubKovÁ B., BARUŠ V., ProkeŠ M. 2005: Associations between fish reproductive cycle and the dynamics of metazoan parasite infection. Parasitol. Res. 95: 65-72.

TER HOfSTEDE H.M., Fenton M.B., WhitAKeR J.O. 2004: Host and host-site specificity of bat flies (Diptera: Streblidae and Nycteribiidae) on Neotropical bats (Chiroptera). Can. J. Zool. 82: 616-626.

WoOTTON R.J. 1976: The Biology of the Sticklebacks. Academic Press, London, $387 \mathrm{pp}$.

WOOTTON R.J. 1984: A Functional Biology of Sticklebacks. Croom Helm, London, 265 pp.

WORTHEN W.B., ROHDE K. 1996: Nested subset analyses of colonization-dominated communities: metazoan ectoparasites of marine fishes. Oikos 75: 471-478.

Zietara M.S., ARndt A., Geets A., Hellemans B., VolCKAERT F.A.M. 2000: The nuclear rDNA region of Gyrodactylus arcuatus and G. branchicus (Monogenea: Gyrodactylidae). J. Parasitol. 86: 1368-1373.

ZiETARA M.S., HuYSE T., LuMME J., VOLCKAERT F.A.M. 2002: Deep divergence among subgenera of Gyrodactylus inferred from rDNA ITS region. Parasitology 124: 39-52.

ZIETARA M.S., LUMME J. 2003: The crossroads of molecular, typological and biological species concepts: two new species of Gyrodactylus Nordmann, 1832 (Monogenea: Gyrodactylidae). Syst. Parasitol. 55: 39-52. 